\title{
Research on Modeling Method of Power System Key Components for E- REV
}

\author{
Jigao Niu${ }^{1, a}$, Xiaoli Shi ${ }^{1}$, Chunhua $\mathrm{Xu}^{1}$ and Chenxu $\mathrm{Li}^{1}$ \\ ${ }^{1}$ Zhongyuan University of Technology, School of Mechanical \& Electronic Engineering, Zhengzhou 450007, China
}

\begin{abstract}
Aiming at the modeling problem of power system key components for an Extended-Range Electric Vehicle (E-REV), the co-simulation platform based on AVL-Cruise and Matlab/Simulink software is introduced, and the modeling methods of E-REV engine, driving motor and battery are studies by the method of mixing the theoretical modeling with the experimental modeling. Off-line simulation results with chosen driving cycles indicate that the proposed vehicle model has better simulation precision and can meet the simulation specification of the E-REV dynamic performance.
\end{abstract}

\section{Introduction}

Through its charging device, the E-REV can get electric energy from power grid. It not just dramatically decreases the human dependency on petroleum, but effectively reduces air pollution. Because of these merits, E-REV has become an alternative development and research direction of clean energy vehicles [1-3]. By building reasonable and effective simulation model of power train components in the process of E-REV development, the effects of power train parameters matching to the vehicle performance can be quantitatively evaluated. Moreover, under the promise of vehicle performance and driving range, the optimal scheme of power train parameters matching can be obtained conveniently and quickly. In addition, the energy management strategy (EMS) of the E-REV can be developed by using the simulation platform, which can verify the advantages and disadvantages of various control strategies quickly, and reduce the unnecessary vehicle manufacture and test.

Firstly, a co-simulation platform of AVL-Cruise and Matlab/Simulink is introduced. Secondly, the modeling methods of the E-REV power train key components including the engine, driving motor and power battery are studied by adopting the way of mixing the theoretical modeling with the experimental modeling. Finally, the model precision is verified by off-line simulation.

\section{E-REV co-simulation platform}

AVL Cruise is a software tool developed by AVL LIST $\mathrm{GmbH}$, which could simulate vehicle dynamics, fuel economy performance and emissions [4]. The graphical modeling method and friendly interface function with Matlab/Simulink make it more popular in the modeling and simulation of traditional automobile and hybrid

\footnotetext{
a Corresponding author: jigaoniulucky@163.com
}

electric vehicle. Cruise model elements include vehicle, battery, motor, engine, clutch, wheels, cockpit, monitor, brakes, electrical components, mechanical components, etc., and using these elements, E-REV simulation platform can be established. Among them, the EMS and fuel economy calculation models are built in Matlab/Simulink software. Figure 1 shows the cosimulation platform of the E-REV.

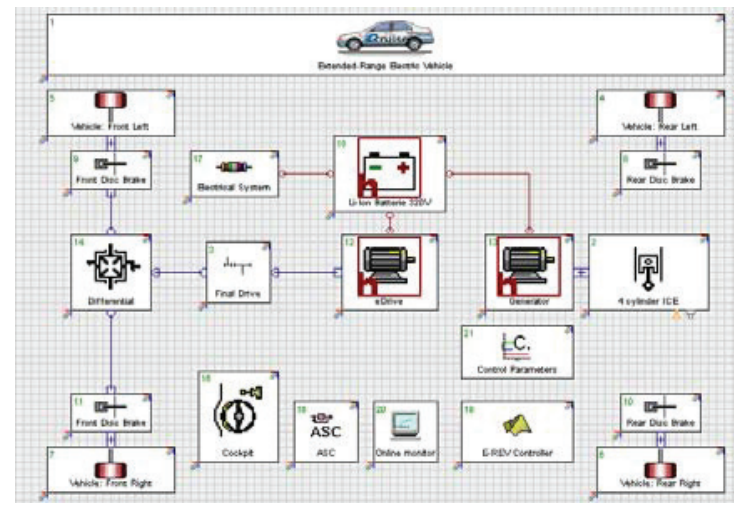

Figure 1. Co-simulation platform of E-REV

\section{Key components modeling of power system}

The key components of the E-REV power system include engine, driving motor and power battery, the modeling method of these components are described as follows.

\subsection{Engine model}

Under the range-extended mode, the engine is the main power source of E-REV, and the engine model accuracy has a great influence on the vehicle performance 
simulation. At present, there are two modeling methods, namely theoretical modeling and experimental modeling. Because the lookup method has a series of advantages, such as simple process, high precision and easy realization, therefore, this paper uses the lookup method to establish the engine model, which includes the engine torque characteristics and throttle opening relationship model, and the engine fuel consumption and emissions model.

\subsubsection{Output torque model}

According to the universal characteristics experimental data of the engine, Figure 2 shows the torque figure of the engine, which describes the engine torque relationship with throttle opening and speed. By using the lookup and interpolation method, the engine output torque can be obtained as

$$
T_{e}=f_{T_{e}}\left(n_{e}, \alpha_{e}\right)
$$

where $T_{e}$ is the engine output torque in $\mathrm{N} \cdot \mathrm{m}, n_{e}$ is the engine speed in rpm, $\alpha_{e}$ is the throttle opening from the vehicle controller unit (VCU).

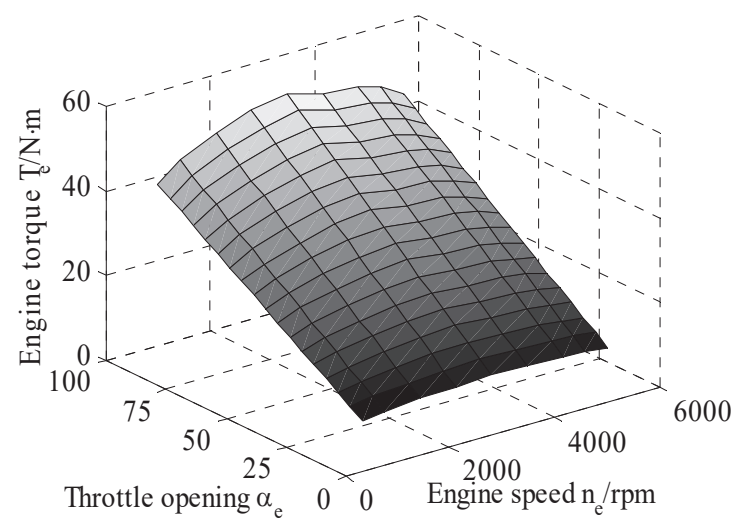

Figure 2. Engine steady torque characteristic

It is well known that the output torque of the engine is decreased because of the friction torque $T_{e f}$, which is increased with the increasing engine speed. Therefore, the friction torque should be considered when the engine is modeled. Based on factors mentioned above, the postcorrection output torque of the engine can be expressed as

$$
T_{e}= \begin{cases}T_{e f}\left(n_{e}\right)+\alpha_{e}\left[T_{e_{-} \max }\left(n_{e}, \alpha_{e}\right)-T_{e f}\left(n_{e}\right)\right], & \alpha_{e} \in[0,1] \text { and } n_{e} \geq n_{\text {idle }} \\ 0, & \text { others }\end{cases}
$$

where $T_{e f}$ and $T_{e_{-} \max }$ are the friction torque and maximum output torque of the engine in $\mathrm{N} \cdot \mathrm{m}$ at the speed $n_{e}$, respectively.

\subsubsection{Fuel consumption model}

Based on universal characteristics experimental data of the engine, the characteristic diagram of the specific fuel consumption is shown in Figure 3, which describes the engine fuel consumption relationship with the engine torque and speed.

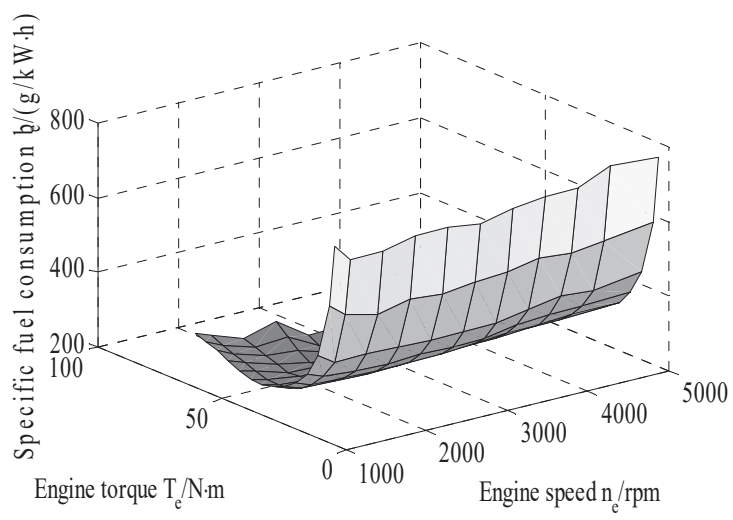

Figure 3. Engine specific fuel consumption characteristic

According to the specific fuel consumption of the engine operating point (Figure 3 ) and the output power, the engine fuel consumption of per hour can be obtained, and then the engine fuel consumption can be calculated by the integral operation. The calculation formulas are given by

$$
\begin{gathered}
b_{e}=f_{b}\left(n_{e}, T_{e}\right) \\
q=\frac{P_{i c e} b_{e}}{1000} \\
Q=\frac{1}{3600 \rho_{\text {fuel }}} \int_{0}^{T} q d t
\end{gathered}
$$

where $b_{e}$ is the engine specific fuel consumption in $\mathrm{g} \cdot(\mathrm{kW} \cdot \mathrm{h})^{-1}, q$ is the fuel consumption of per hour in $\mathrm{kg} \cdot \mathrm{h}^{-}$ ${ }^{1}, P_{\text {ice }}$ is the output power of the engine operating point in $\mathrm{kW}, Q$ is the fuel consumption of the engine in $\mathrm{L}, \rho_{\text {fuel }}$ is the fuel density in $\mathrm{kg} \cdot \mathrm{L}^{-1}$, and $T$ is the running time in $\mathrm{s}$.

Simultaneously, according to the parameters of $P_{\text {ice }}$ and $q$, the efficiency of the engine operating point can be calculated as

$$
\eta_{e}=\frac{3600 P_{i c e}}{q H_{\text {fuel }}}
$$

where $H_{\text {fuel }}$ is the fuel heat value in $\mathrm{KJ} \cdot \mathrm{kg}^{-1}$.

The modeling methods of the engine exhaust such as $\mathrm{CO}, \mathrm{HC}$ and $\mathrm{NO}_{x}$ are similar to the fuel consumption as discussed above.

\subsection{Motor model}

The motor model adopts generally the theoretical model or the quasi-steady model based on the motor efficiency chart, but the former needs to obtain the motor physical parameters, and the simulation speed is slower, therefore, it is generally used to evaluate the motor dynamic characteristics. The latter is the most commonly used in the motor model, which is similar to the engine model, and its advantage is that when the accurate motor physical parameters cannot obtained, the motor performance can be intuitively evaluated according to the motor characteristics parameters. Therefore, it is suitable for various types of motor models. 
Based on the experimental data of the motor external characteristic, Figure 4 shows the 3D curved surface figure, which describes the relationship of the motor steady torque measured with the load signal (i.e., motor throttle signal) and the motor speed. The output steady torque of the motor can be obtained by using the lookup method. Moreover, the motor output steady torque can be modified by introducing one order inertial link, and the dynamic output torque of the motor is given by the following equations.

$$
\begin{aligned}
& T_{m_{-} s}=f_{T_{m_{-} s}}\left(n_{m}, \beta_{m}\right) \\
& T_{m}=T_{m_{-} s} \frac{1}{K_{t} s+1}
\end{aligned}
$$

where $T_{m_{-} s}$ and $T_{m}$ are the output steady torque and dynamic torque of the motor in $\mathrm{N} \cdot \mathrm{m}$, respectively, $n_{m}$ is the motor speed in rpm, $\beta_{m}$ is the load signal of the motor from the VCU in $\%, K_{t}$ is the time constant, and $S$ is the laplace transform factor.

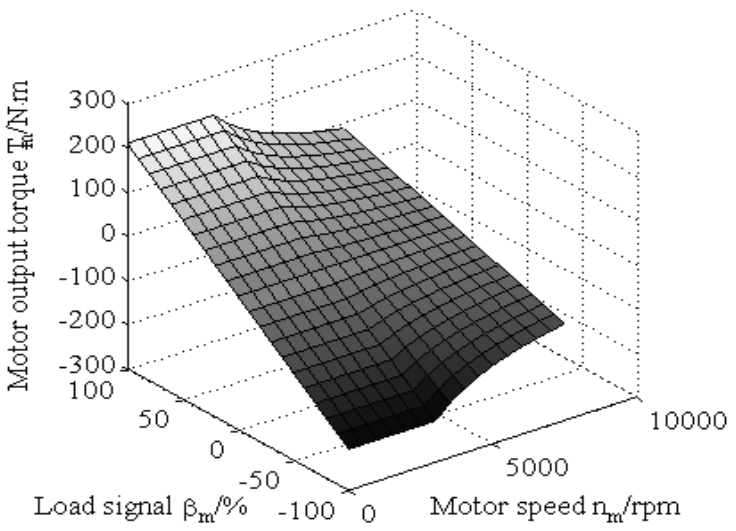

Figure 4. Motor steady torque characteristic

According to the experimental data of the motor efficiency characteristic, Figure 5 shows the motor efficiency relationship with the motor speed and torque.

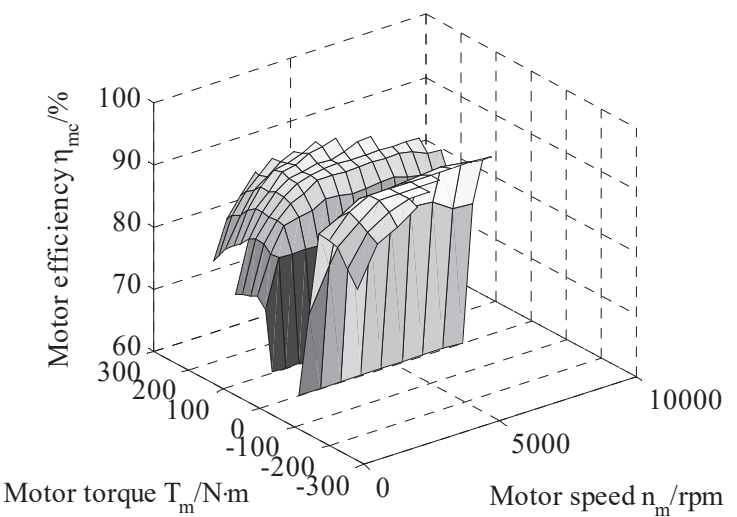

Figure 5. Motor efficiency characteristic

By the way of lookup, the motor efficiency $\eta_{m c}$ can be expressed as

$$
\eta_{m c}=f_{m_{-} \eta}\left(n_{m}, T_{m}\right)
$$

From Equations 8 and 9, the motor current is calculated by using the method of lookup and interpolation as follows
$I_{m_{-} \text {net }}=\left\{\begin{array}{l}\left(1000 T_{m} n_{m}\right) /\left(9550 \eta_{m c} U_{m_{-} n e t}\right), \text { electric mode } \\ \left(1000 T_{m} n_{m} \eta_{m c}\right) /\left(9550 U_{m_{-} n e t}\right), \text { generating mode }\end{array}\right.$

where $I_{m \_n e t}$ is the motor current in A, $U_{m_{-} \text {net }}$ is the motor voltage in $\mathrm{V}$.

The effective output torque of the motor is given by

$$
T_{m_{-} \text {out }}=T_{m}-J_{m} \omega_{m}
$$

where $J_{m}$ is the rotational inertia of the motor rotor in $\mathrm{kg} \cdot \mathrm{m}^{2}, \omega_{m}$ is the motor angular acceleration in $\mathrm{rad} \cdot \mathrm{s}^{-2}$.

\subsection{Battery model}

The battery charge-discharge is associated with the complex electrochemical reaction, and its performance parameters are greatly affected by temperature, consistency and other factors, therefore, it is difficult to describe the dynamic characteristics of the power battery by the accurate mathematical model [5]. There are two equivalent circuit models (i.e. Rint and RC) in Cruise, and Rint model is adopted in this paper. In this model, the battery is equivalent to an ideal voltage source $U_{o c v}$, which is series with the battery internal resistance $R_{\text {batt }}$. Rint circuit model is shown in Figure 6.

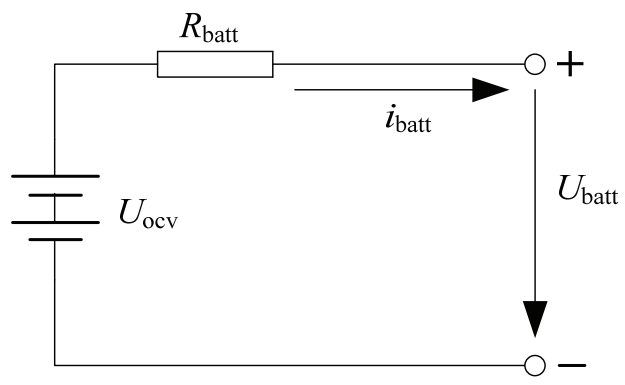

Figure 6. Equivalent circuit of Rint model

According to the Kirchhoff Law, the output voltage of the battery model (see Figure 6) can be written as

$$
U_{\text {batt }}=U_{\text {ocv }}-i_{\text {batt }} R_{\text {batt }}
$$

where $i_{\text {batt }}$ is the battery charge and discharge current in A, $R_{\text {batt }}$ is the battery charge and discharge internal resistance in $\Omega$.

The state of charge (SOC) of the battery reflects its capacity state, and the SOC can be expressed as

$$
S O C=C_{r} / C_{I_{-} \max }
$$

where $C_{r}$ and $C_{I_{-} \max }$ are the residual capacity and actual capacity of the battery when discharging with the constant current in $A \cdot h$, respectively.

According to the total capacity $C_{I \max }$ and initial capacity $C_{i}$ of the battery, the input or output electric quantity $C_{r e}$ can be calculated by using the adding method. The current SOC is given by the equations (14) (16).

$$
C_{i}=S O C_{i} C_{I_{-} \max }
$$




$$
\begin{aligned}
& C_{r e}=\frac{1}{3600} \int_{0}^{t} i_{b a t t} d t \\
& S O C=\frac{C_{i}-C_{r e}}{C_{I_{-} \max }}
\end{aligned}
$$

where $C_{i}$ is the initial SOC, $i_{\text {batt }}$ is the charge and discharge current in A, $t$ is the charge and discharge time in $\mathrm{s}$, the unit of the battery capacity and electric quantity is $\mathrm{A} \cdot \mathrm{h}$.

The battery discharge efficiency is represented with the energy efficiency $\eta_{\text {batt }}$, which is the ratio of the discharge total energy $E_{T, I}$ and the nominal energy $E_{n}$. $\eta_{\text {batt }}$ can be expressed as

$$
\eta_{\text {batt }}=\frac{E_{T, I}}{E_{n}} \times 100 \%
$$

where $\eta_{\text {batt }}$ is the discharge energy efficiency of the battery, $E_{n}$ is the nominal energy of batteries in $\mathrm{W} \cdot \mathrm{h}$, and $E_{T, I}$ is the total discharge energy of the battery under the condition of temperature $T$ and current $I, \mathrm{~W} \cdot \mathrm{h}$.

In order to get the battery experimental data, the $\mathrm{LiFePO}_{4}$ power battery pack $(320 \mathrm{~V} / 50 \mathrm{~A} \cdot \mathrm{h})$ is taken as the testing object in this paper. The open circuit voltage $(\mathrm{OCV})$ and charge-discharge internal resistance of the battery are tested at normal temperature, respectively. Moreover, the hybrid pulse power characterization is used in the test for the charge-discharge internal resistance.

Figure 7 shows the SOC-OCV curve, which is obtained in the process of the battery charging and discharging at normal temperature. From Figure 7, it can be observed that the OCV curve is not coincident, and the charging curve is always slightly higher than the discharging.

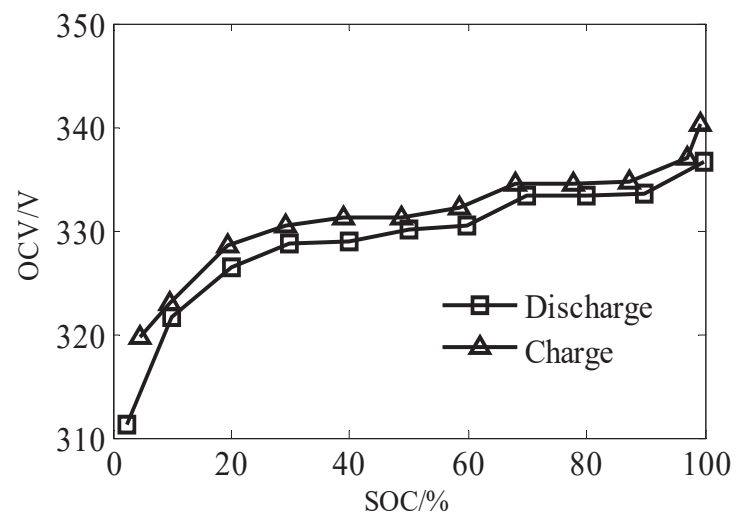

Figure 7. OCV -SOC charging and discharging curve $\left(25^{\circ} \mathrm{C}\right)$

Figure 8 and Figure 9 show the relationships between the internal resistance of the discharge and charge with the SOC at normal temperature, respectively. It can be seen that when the SOC is located at low region, the internal resistance of the battery discharge and charging is increased drastically, and in other relatively wide SOC ranges, the internal resistance curve of the battery becomes gentle.

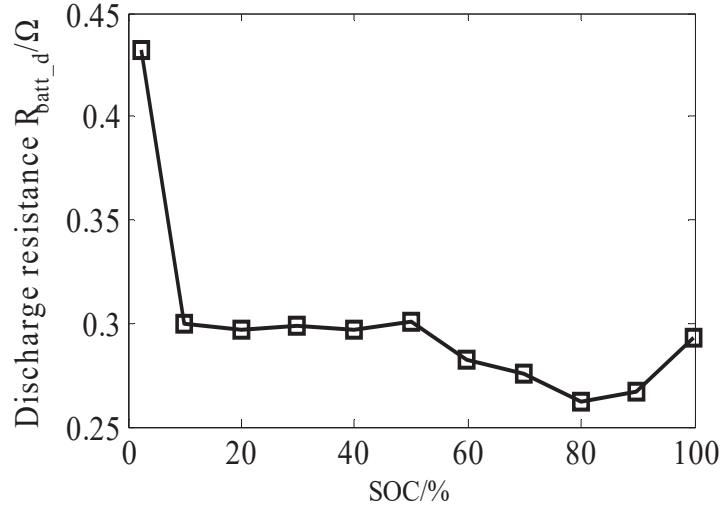

Figure 8. Battery discharge resistance curve

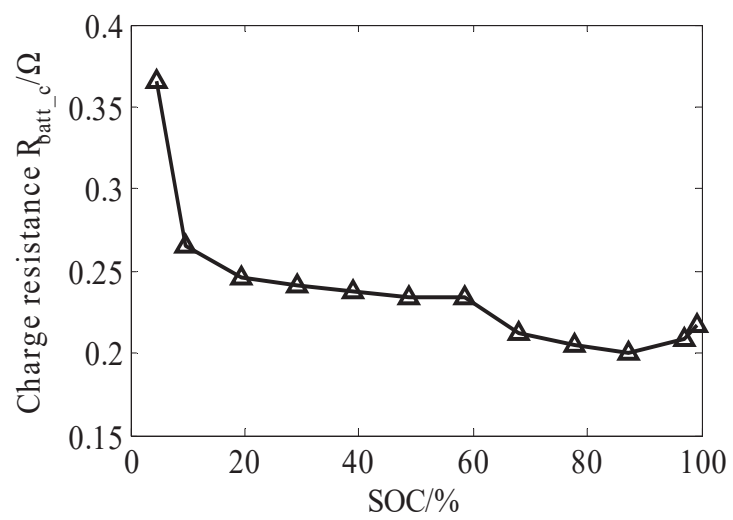

Figure 9. Battery charge resistance curve

\section{Simulation results analysis}

In order to verify the model precision, the vehicle data and model mentioned above are embedded into the EREV simulation platform and the simulation calculation of the dynamic performance with a chosen driving cycle are conducted. Figure 10 shows the vehicle speed simulation result in an NEDC drive cycle, and Figure 11 shows the tracking error of the vehicle speed. Off-line simulation results indicate that the simulation speed can follow the driving cycles speed appropriately, and the parameters matching of the E-REV power train can meet the requirements of speed and acceleration. Therefore, the model precision of the key components above mentioned can meet the design requirements.

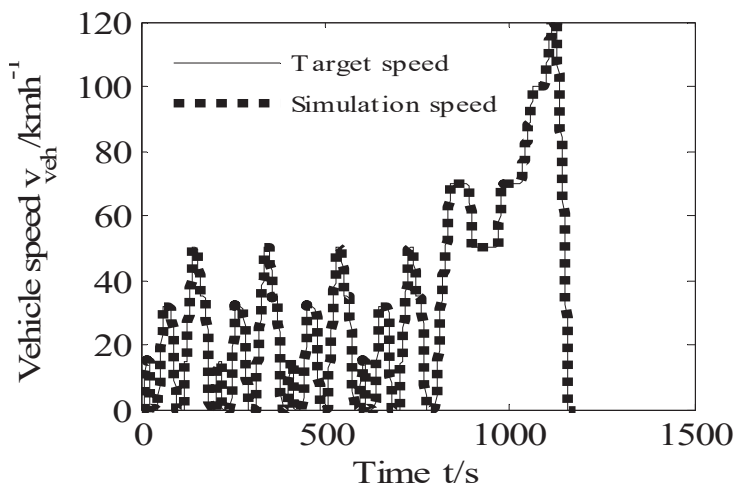

Figure 10. Results comparison of vehicle speed 


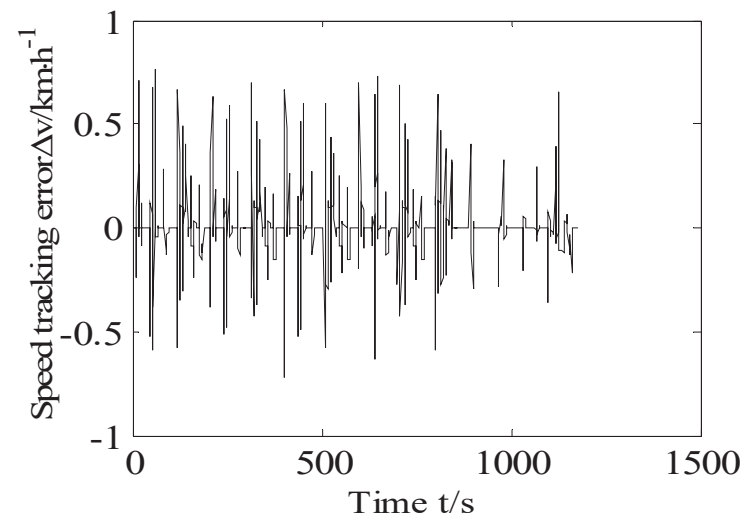

Figure 11. Tracking error of vehicle speed

\section{Conclusion}

The modeling methods of E-REV power train key components including the engine, driving motor and power battery are studied by adopting the way of mixing the theoretical modeling with the experimental modeling in this paper. Off-line simulation results with chosen driving cycles indicate that the proposed vehicle model has better simulation precision and can meet the simulation specification of the E-REV dynamic performance.

\section{Acknowledgment}

The work is supported by Key Research Projects of Henan Higher Institution "Optimization and Control Research of Energy Management Strategy for E-REV" (Item Number: 17A460006).

\section{References}

1. G. Fontaras, Z. Samaras. A quantitative analysis of the European automakers' voluntary commitment to reduce $\mathrm{CO}_{2}$ emission from new passenger cars based on independent experimental data. Energy Policy. J. 35 2239-2248 (2007)

2. J Guanetti, S Formentin, S M. Savaresi. Energy management system for an electric vehicle with a rental range extender: a least costly approach. IEEE T INTELL TRANSP. J. 17, 3022-3034(2016)

3. Y. Wang, B. Meng, Y P. Shen, et al. Researches on power systems of extended range electric vehicles. Proceedings of the CSEE. J. 34, 4629-4639 (2014). (in Chinese)

4. S. Yang, B. Qiu, L. Ta, et al. A Study on Simulation of Energy Management Strategy of Extended Range Hybrid Bus Based on Cruise. Automobile Technology. J.7 37-42 (2017) (in Chinese)

5. L. Ramotar, G L. Rohrauer, R. Filion, et al. Experimental verification of a thermal equivalent circuit dynamic model on an extended range electric vehicle battery pack [J]. Journal of Power Sources, J 343, 383-394 (2017) 